\title{
LA CALIDAD DE VIDA INFANTIL Y SUS DIMENSIONES: PERCEPCIÓN DE ESCOLARES
}

\author{
$*$ \\ Lia Machado Fiuza Fialho \\ Universidade Estadual do Ceará - UECE - Brasil \\ José Rogério Santana \\ Universidade Estadual do Ceará - UECE - Brasil \\ Cristine Brandenburg \\ Universidade Estadual do Ceará - UECE - Brasil
}

\section{Resumen}

El estudio pretende evaluar la Calidad de Vida (CV) percibida de escolares, identificar la relevancia de sus dimensiones desde la óptica de niños de escuela pública y particular en Fortaleza-CE, y determinar si la dimensión Rendimiento Escolar se relacionaba con la CV. Participaron 90 niños, entre ocho y once años, que realizaron dos tareas: contestar al cuestionario AUQEI (Manificat \& Dazord, 1997) y efectuar el ordenamiento de dimensiones de CV. También fue correlacionado el rendimiento escolar com la puntuación final del AUQEI. Fue comprovado que la CV de los niños fue satisfactoria aunque con diferencia significativa a favor de los niños de la escuela particular, las dimensiones más relevantes para todos los escolares fueron: Rendimiento, Bienestar Físico y Emocional y Relaciones Interpersonales, con diferencias al analizar las preferencias por tipo de escuela; no hubo relación entre Rendimiento escolar y el puntaje final de CV.

Palabras clave: Calidad de vida. Niño. Escuela.

\section{Indroducción}

Calidad de Vida (CV) es una noción eminentemente humana y abarca varios significados que reflejan conocimientos, experiencias y valores de individuos y colectividades. Tales significados reflejan el momento histórico, la clase social y la cultura a que pertenecen los individuos (MINAYO et. al, 2000; DANTAS, SAWADA, MALERBO, 2003). Schalock y Verdugo (2003) acentúan que los conceptos y modelos de CV cambian potencialmente de país para país e incluso de región para región dentro de un mismo país.

Desde una perspectiva histórica, los estudios sobre CV infantil son menos numerosos que en adultos (EISER, MORSE, 2001) y han sido realizados predominantemente en el campo de la Calidad de Vida relacionada a la Salud (CVS), investigando niños con 
enfermedades crónicas, aunque aún no forman parte de las prácticas clínicas de rutina (EISER, EISER, STRIDE, 2005).

Calidad de Vida infantil ha sido definida como un concepto subjetivo y multimensional que incluye la capacidad funcional y la interacción social del niño y su familia (BRASIL, FERRIANI, MACHADO, 2003). Otros autores, coinciden en su multidimensionalidad y señalan que abarca varios dominios centrales, como condiciones materiales, condición física y habilidades funcionales, interacción social y bienestar emocional (WHITE-KONING M, et al, 2005). Ambas definiciones provienen de estudios que evalúan niños con discapacidad, desde un enfoque de CVS.

Los estudios de CV en el contexto escolar son aún menos frecuentes que en CVS. Los primeros trabajos en este sentido fueron realizados en la década del 70 (EPSTEIN, MC PARTLAND, 1976), perdurando todavía la discusión en la psicología educacional sobre las fronteras entre el constructo CV y otras categorías (MOK, MCDONALD, 1994). En la actualidad, aún se consideran incipientes los trabajos que analizan la Calidad de Vida percibida por el escolar y la influencia que la escuela puede ejercer en esa CV.

Según Gómez-Vela y Verdugo (2003), el uso de este concepto en la escuela puede traer una serie de beneficios: avanzar en el desarrollo integral del alumno, como referencia y noción sensibilizadora para la planificación y gestión que la escuela necesita para los estudiantes y la comunidad educativa en general, con modelos centrados en la persona y para detectar desigualdades y grupos de riesgo.

Tradicionalmente los niños han sido considerados como no confiables para responder acerca de su propia CV, de forma que ha predominado la tendencia a utilizar a adultos (padres o cuidadores, profesionales que los atienden) como fuente de informaciones, reconociendo sin embargo que pueden existir diferencias entre lo que es CV desde la visión de un adulto y del propio niño (ASSUMPÇÃO et. al, 2000; EISER, 2001; JOKOVICH, 2004). Este hecho ha despertado un creciente interés por oír al propio niño, sus opiniones y criterios, lo que metodológicamente se refleja en enfoques de evaluación que privilegian y fortalecen al niño como participante, en el surgimiento de un mayor número de pruebas a ser respondidas por el niño y al incremento de estudios cualitativos (REJMIL, 2004; HARDING, 2001; PREBIANCHI, 2003; SABEH, VERDUGO, PRIETO, 2006)

Buscando identificar cuáles eran las dimensiones de Calidad de Vida relevantes para los niños en Argentina y España, Verdugo \& Sabeh (2002) desarrollaron un estudio inicial, 
de tipo cualitativo en el cual participaron 199 niños de 8 a 12 años, que permitió identificar las siguientes dimensiones, expresadas de la siguiente forma:

1- Ocio y actividades recreativas (satisfacción, insatisfacción o deseos de mejora expresados por el niño en experiencias de ocio, recreativas y de tiempo libre tales como juegos, deportes, actividad física, televisión, vídeos, realizados de forma individual o colectiva);

2- Rendimiento (satisfacción, insatisfacción o deseos de mejora expresados por el niño en relación al desempeño y los resultados alcanzados en actividades escolares, deportivas);

3- Relaciones Interpersonales: (satisfacción, insatisfacción o deseos de mejora expresados por el niño en relación a la interacción positiva o negativa (conflictos) con y entre personas del medio familiar, escolar, amigos, conocidos, así como las actitudes positivas o negativas y la presencia o ausencia de los mismos, incluyendo en esta dimensión el vínculo con animales);

4- Bienestar físico y emocional : satisfacción, insatisfacción o deseos de mejora expresados por el niño en relación a su estado físico o de salud, así como al de sus familiares o seres queridos, así como sus apreciaciones o preocupaciones con el bienestar general de los mismos);

5- Bienestar Colectivo y Valores (satisfacción, insatisfacción o deseos de mejora que expresa el niño en relación a la situación social, económica y política, que percibe del medio sociocultural en el cual vive, así como en relación a valores humanos)

6- Bienestar material (vinculada a la consecución y relación con objetos (regalos, por ejemplo) y a las características de los ambientes en los que se desarrolla.

Una propuesta de conceptualización, que considera el estudio antes descrito, define a la Calidad de Vida en la infancia como la percepción subjetiva del niño en conjunción con aspectos objetivos referidos a cinco dominios: Desarrollo Personal y Actividades, Relaciones Interpersonales, Bienestar Físico, Bienestar Emocional y Bienestar Material, (SABEH et al, 2003; SABEH, VERDUGO, PRIETO, 2006). La definición del contenido de los dominios establecidos en 2003 permite apreciar las relaciones con las dimensiones del estudio inicial de 2002, apreciándose que Ocio y Actividades Recreativas y Rendimiento pasaron a formar parte del dominio Desarrollo Personal y Actividades mientras que Bienestar Físico y Emocional se dividió en dominios independientes.

En Brasil, los estudios sobre calidad de vida infantil en el contexto escolar están en fase inicial. Un estudio realizado por Dantas, Sawada y Malerbo (2003) analizó la producción científica sobre Calidad de Vida, de universidades públicas con amplia tradición de investigación del estado de São Paulo. Este estudio fue de tipo exploratorio descriptivo, con datos colectados a través de bibliotecas virtuales de las universidades escogidas. Fueron 
analizados 53 estudios, siendo 29 (54,7\%) provenientes de la Universidad de São Paulo (USP) y $24(45,3 \%)$ de la Universidad Federal de São Paulo (UNIFESP). Sólo un trabajo analizó la Calidad de Vida de niños, específicamente en relación con la salud.

En estudio de revisión sistemática realizado por Fialho y Valdés (2006), analizando la producción científica registrada en la base de datos Scielo Brasil, que agrupa las principales revistas brasileras en todas las áreas del conocimiento, en el período 2000-2007, con los descriptores qualidade de vida, infância, y escola, se encontraron 4 trabajos referidos a Calidad de Vida relacionada a la salud, 1 trabajo de adaptación de un instrumento genérico y no fue identificado ningún trabajo que analizara Calidad de Vida en la escuela pues los que se clasificaban en estos descriptores realmente analizaban Calidad de Vida relacionada a la salud, o sea, influencia de determinada enfermedad en la vida de escolares.

Considerando las seis dimensiones de CV infantil definidas en el estudio inicial de Verdugo y Sabeh (2002), se desarrolló un estudio de evaluación de calidad de vida en la escuela con niños brasileros de 8 a 11 años que cursan la tercera serie de la enseñanza fundamental I, con el objetivo de identificar las dimensiones más relevantes de calidad de vida en la perspectiva del propio niño, averiguar la posible relación entre la dimensión de Rendimiento y la puntuación final de calidad de vida de los educandos y determinar las posibles diferencias entre alumnos de escuela pública y privada.

\section{Participantes}

Participaron noventa niños y niñas, con edad entre ocho y once años, con media de edad de 9,41 años, todos los que estaban matriculados en la tercera serie de la enseñanza fundamental I de dos escuelas, una pública y otra particular, del municipio de Fortaleza, Ceará, Brasil, en las que se realizó la investigación. Fue determinado este grupo de edad por el hecho de que de los 8 a los 11 años son considerados los últimos años de la infancia y niños de estas edades tienen mayor desarrollo del autoconcepto y de la autoestima, capacidad de evaluar suas prpias capacidades y competencias (PALACIOS, HIDALGO, 2004), lo que facilita la autoevaluación de su Calidad de Vida.

Fueron 47 niños y 43 niñas, de los cuales 24 y 21 respectivamente pertenecían a la escuela pública y 23 y 22 a la particular. No participaron únicamente aquellos niños que aunque manifestaron su deseo de responder, sus padres (o responsables) no firmaron el Término de consentimiento libre y esclarecido. 
Entre los participantes, sólo uma niña de la escuela particular había sido evaluada y diagnosticada como Necesidad Educativa Especial, de tipo Discapacidad auditiva media, mas utilizava aparatos auditivos bilaterales y se comunicaba oralmente, lo que posibilitó su participación sin necesidad de procedimientos diferenciados de los otros niños provenientes de esta escuela.

Ambas escuelas poseían una buena infraestrutura física: laboratorio de informática relativamente actualizado, biblioteca con libros variados y accesible a los niños, aulas aireadas, amplias y bien iluminadas, áreas polideportivas, área de ócio y entretenimiento, bebederos, servicios sanitarios higiénicos y en número suficiente, entre otras. No obstante, la escuela particular, dispone de privilegios estructurales que la sitúan por encima de la escuela pública pues tiene una área mucho más amplia, juguetes y equipos más modernos, mayor número de áreas deportivas y ordenadores, etc.

En la selección de las instituciones se tomó em cuenta que estuvieran situadas en el mismo barrio (urbano, populoso) y que tuvieran la infraestructura y abertura necesarias al adecuado desarrollo de la investigación.

Los niños que asisten a la escuela pública son de famílias con un poder adquisitivo considerado bajo, inferior a tres salarios mínimos, no teniendo condiciones de adquirir lo mínimo necesario para una vida confortable. Por lo contrario, los niños que asisten a la escuela particular son considerados de clase media, sus famílias generalmente poseen una renta mayor que tres salários mínimos que posibilita atender a las necesidades básicas y pagar una matrícula mensual de aproximadamente um salario mínimo por cada hijo matriculado en la institución.

La investigación cumplió las exigencias éticas y siguió las normas de la resolución 196/96 del Consejo Nacional de Salud de Brasil, recibiendo evaluación favorable del Comité de Ética en Pesquisa Envolviendo Seres Humanos de la Universidad de Fortaleza. Fue utilizado un Término de Consentimiento Informado, que fue firmado por los niños y niñas participantes y por sus padres o responsables; también fue autorizado el uso de la imagen de 5 niños y niñas en fotografías y las investigadoras asumieron el compromiso de ser fieles depositarias de datos relacionados con el rendimiento escolar de los participantes, obtenidos de su expediente académico en cada escuela.

\section{Métodos}

Para la identificación de las dimensiones más relevantes de calidad de Vida se adoptaron las definiciones del estudio inicial de Verdugo e Sabeh (2002). Considerando las 
recomendaciones de uso de imágenes para estudiar CV en la infancia (MANIFICAT, DAZORD, 1997; EISER, 2001) y por las dificultades reconocidas de comprensión lectora de los niños de escuela pública brasilera, se decidió ilustrar las dimensiones mediante el uso de fotos especialmente creadas para ese fin, escenificadas por niños de la misma edad, en ambientes neutros, o sea, que pudieran representar la dimensión sin referencias socioeconómicas. Así, fueron creadas dieciocho fotos, siendo tres para cada dimensión, que fueron sometidas a técnica de consenso entre expertos, lo que permitió obtener seis fotos donde cada una representa una dimensión; a cada foto le fue colocada una etiqueta con el nombre de la dimensión que representa y su definición. La consigna para la realización de la tarea fue la siguiente "Estas 6 fotos representan dimensiones de calidad de vida. Te pedimos que las organices en un orden que muestre cuál es la más importante para tu vida, la que te trae más felicidad, de la más importante a la menos importante."

Para cada foto, fue establecida una puntuación específica que varía de 6 a 1, de acuerdo con el orden en que el niño coloca la foto. Se otorgó puntuación 6 a la más relevante para su vida y consecutivamente, en orden decreciente, hasta puntuación 1 a la menos significativa.

Para averiguar la dimensión Rendimiento, por su relevancia en esta edad, se optó por el Rendimiento Escolar, y, como indicador objetivo, se tomó el registro escolar del niño matriculado en la escuela en forma de puntuación anual establecida numéricamente en forma de notas. Esta puntuación individual fue correlacionada con el puntaje final de CV de cada niño.

Se haz necesario distinguir que la evaluación de la CV fue realizada mediante el uso del AUQEI (Autoquestionnaire Qualité de Vie Enfant Imagé), desarrollado en 1997 por Manificat e Dazord en Francia y validado en Brasil en 2000 por Assumpção Jr. et. al. Este instrumento es genérico y posee reconocida validez (DAVIS et. al, 2006).

El cuestionario posee 26 ítems, en que 18 están contenidos en 4 dimensiones básicas: Función (cuestiones relativas a las actividades en la escuela, a las comidas, al acostarse y a la ida al médico) con cinco ítenes $1 ; 2 ; 4 ; 5 ; 8$; Familia (cuestiones relativas a figuras parentales y a los propios niños) con cinco ítenes $3 ; 10 ; 13 ; 16 ; 18$; Tiempo Libre (cuestiones relativas a vacaciones, cumpleaños, y relaciones con los abuelos) con tres ítens 11;21;25; Autonomía (cuestiones relacionadas a la independencia, relación con los compañeros y evaluación) con cinco ítens $15 ; 17 ; 19 ; 23 ; 24$. Para cada pregunta existen cuatro opciones de respuestas: muy infeliz, infeliz, feliz y muy feliz. Cada una de las respuestas tiene una puntuación específica que va de 0 a 3, en una escala ordinal, correspondiendo 0 a Muy Infeliz, lo que posibilita la 
obtención de una puntuación única, resultante de la sumatoria de los puntos atribuídos a los ítems, con una variabilidad de 0 a 78. Cuanto mayor sea la puntuación obtenida, mejor será la Calidad de Vida del niño.

A los efectos de este artículo, sólo se utilizará la puntuación final de calidad de vida, cuyo punto de corte para niños brasileros fue determinado en 48 , indicando por debajo de ese número que la CV está comprometida (ASSUMPÇÃO JR. et al, 2000).

\section{Procedimientos}

La colecta de datos se realizó en los meses de abril y mayo de 2007 en el contexto escolar. La primera tarea era la realización del AUQEI, la segunda el ordenamiento de las dimensiones com auxilio de fotos y finalmente, correlacionar el rendimiento escolar del niño (obtenido de su histórico) con la puntuación de calidad de vida obtenida mediante el AUQEI.

Inicialmente se realizó un estudio piloto en ambas escuelas, en abril del 2006, constatando que en la escuela pública $57 \%$ de los alunos no tenían las habilidades de comprensión lectora y de legilibilidad de la escritura que garantizaran que el AUQEI fuera cumplimentado como cuestionario autoaplicable. Por tanto, en la escuela pública la primera tarea (la explicación y llenado del cuestionario), para esos alumnos, fue realizado de forma individual por una de las investigadoras, lo cual está previsto en la prueba, sólo que para niños de menor edad.

En la escuela particular, el cuestionario AUQEI, fue explicado con auxilio de las cuatro imágenes de la prueba y posteriormente realizado integralmente por los niños de forma colectiva con la presencia de una de las investigadoras em la sala para aclarar dudas siempre que se hiciera necesario. Todos los niños respondieron el cuestionario, comenzando con las respuestas abiertas a las cuatro preguntas relacionadas a lo que los dejaba "muy infeliz", "infeliz", "feliz" y "muy feliz" y posteriormente marcando con un X una de las cuatro opciones presentadas frente a cada situación expuesta en los 26 itens de la prueba.

Segunda tarea: Los alumnos fueron convidados a organizar las dimensiones individualmente, colocándolas en orden de importancia para su vida, quedando en primer lugar la que consideró más relevante y respectivamente, en último, la que consideró menos relevante.

Los datos obtenidos del AUQEI fueron puntuados mediante la suma de puntajes, de la forma prevista en la prueba. El análisis descriptivo y analítico del estudio fue realizado mediante el programa estadístico SPSS (Statistical Package for the Social Sciences). La 
normalidad de las variables fue testada utilizando la prueba Kolmogorov-Smirnov, que demostró una distribución anormal. La prueba $\mathrm{T}$ simple independiente fue utilizada para comparar los puntajes de la escuela pública y privada em las diferentes dimensiones de CV. Fue analizada la correlación entre Rendimiento Escolar y Puntaje Final de Calidad de Vida mediante coeficiente de correlación de Spearmann.

\section{Resultados}

Se constató que, de una variación posible de 0 a 78, la puntuación media total de Calidad de Vida (ECV) fue de 51,12 para todos los 90 niños de la muestra. No obstante, los pertenecientes a la escuela particular obtuvieron mejor ECV $(52,67)$ que los provenientes de la escuela pública $\mathrm{ECV}=49,57$, a pesar de que en ambos casos presentaron una puntuación media de Calidad de Vida satisfactoria, como puede observarse en la tabla 1.

Tabla 1 -Calidad de Vida (CV) por Escuela

\begin{tabular}{|l|l|l|ll|l|}
\hline \multicolumn{2}{|c|}{} & Escuela Pública & $\begin{array}{l}\text { Escuela } \\
\text { Particular }\end{array}$ & Total \\
\hline \multirow{4}{*}{ AUQEI } & Media CV & 49,57 & 52,67 & 51,12 \\
\cline { 2 - 7 } & \multicolumn{7}{|l}{} & \\
\cline { 2 - 7 } & $<48$ & $17(37,7)$ & 8 & $(17,7)$ & 25 \\
\cline { 2 - 7 } & 248 & $28(62,2)$ & 37 & $(82,2)$ & 65 \\
\cline { 2 - 7 } & Total de Niños & 45 & 45 & 90 \\
\hline
\end{tabular}

Fuente: Cuestionarios AUQEI

La puntuación media de CV general de los dos grupos mostró una diferencia estadísticamente significativa, ya que mientras que en la escuela particular apenas $8(17,7 \%)$ de los 45 alumnos presentaron $\mathrm{ECV}<48$, en la escuela pública ese número se duplicó, pasando para $17(37,7 \%)$ alumnos con $\mathrm{ECV}<48$.

Después del cálculo de las puntuaciones totales de calidad de vida se inició la tabulación de las dimensiones de CV más significativas desde la óptica del niño. Se constató que el orden de las dimensiones fue respectivamente: Rendimiento (72,2 \%), Bienestar Físico e Emocional (67,0\%), Relaciones Interpersonales (61,5\%), Bienestar Colectivo y Valores (54,4\%), Actividades Recreativas (52,0\%) y Bienestar Material (42,8\%), como puede observarse en el Gráfico 1. 
Gráfico 1 - Relevancia de las Dimensiones de Calidad De Vida según grupo.

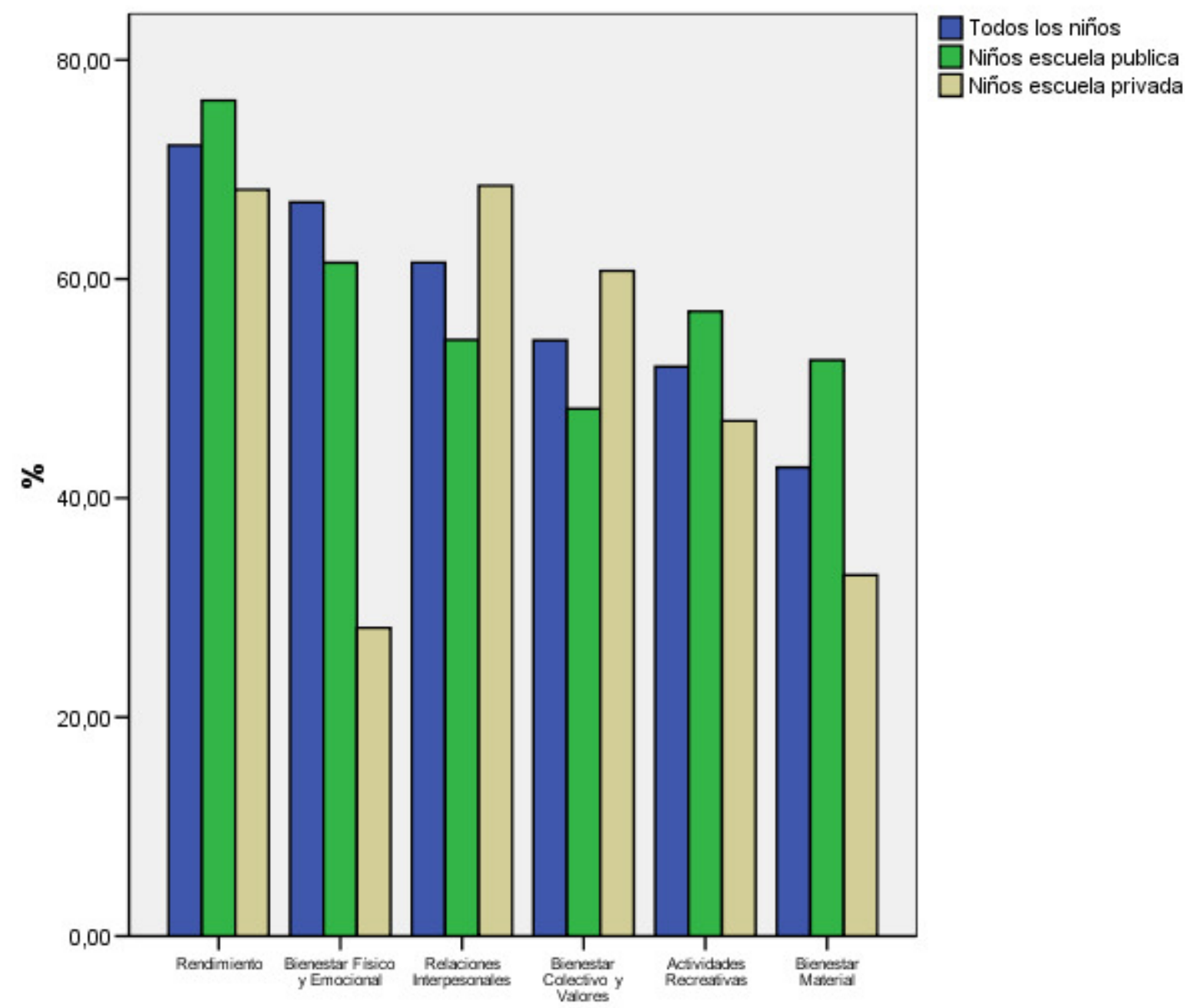

Sin embargo, es importante observar la diferencia en la escala de relevancia entre la escuela pública y la escuela privada cuando son analizadas separadamente. El porcentaje que cada dimensión asume es diferente y el orden de importancia atribuido a las dimensiones varía según el tipo de escuela.

Fue constatado que las dimensiones más relevantes para los alumnos de la escuela pública fueron: Rendimiento (76,30\%), Bienestar Físico y Emocional (61,48\%), Actividades Recreativas (57,04\%), Relaciones Interpersonales (54,44\%), Bienestar Material (52,59\%), Bienestar Colectivo y Valores (48,15\%). Estos resultados divergen de los encontrados desde la visión de los niños de la escuela particular., que priorizaron la dimensión Relaciones Interpersonales $(68,52 \%)$, posteriormente citaron el Rendimiento $(68,52 \%)$, el Bienestar Colectivo y Valores $(60,74 \%)$ que en la escuela pública se ubicó en la última posición, 
seguida de Ocio y Actividades Recreativas (47,04\%), Bienestar Material (32,95\%) y Bienestar Físico e Emocional (28,15\%).

Cuando se analizó la posible relación entre Rendimiento Escolar de los alumnos y el puntaje final de calidad de vida, se obtuvo un rs de 0,174 y p=0,89, el que indica que no existió correlación entre ambos.

\section{Discusión}

Como observado en la tabla 1, los valores medios de Calidad de Vida para las dos escuelas son satisfactorios. En estudio realizado por Asumpção Jr et. al. (2000), durante el proceso de validación del AUQEI, el cuestionario fue aplicado a 353 niños de clase media de la ciudad de São Paulo, encontrando una media de puntuación de calidad de vida ECV= 52,1, valor similar al encontrado en los niños de la escuela particular (52,67). El ECV de los niños de escuela pública es significativamente diferente $(49,57)$ de estos valores y aún demostrando una calidad de vida satisfactoria, se mostró menor que el referido valor.

Considerando que Calidad de vida es un constructo multidimensional y que los niños de escuela pública son reconocidos como una población de baja renta, residentes en la favela del barrio, que vivencian situaciones precarias de saneamiento básico (abastecimiento de agua, recogida de desechos sólidos y red de alcantarillado), limitaciones diversas en el acceso a los servicios de salud y de educación y baja calidad de los servicios que reciben, ausencia de bienes materiales importantes como casa, alimentación, vestuario y otros, podría ser inferido que estos factores interfieren de forma negativa en su calidad de vida en general.

Las graves condiciones de la escuela pública en Brasil, fundamentalmente en la educación básica, representan un reto para los profesionales que en ella actúan, incluyendo a pedagogos y psicólogos educacionales (GUZZO, 2003) mas la escuela también representa para muchos niños el lugar donde encontrar la principal fuente de alimentación diaria, de juguetes y libros.

El orden de relevancia de las dimensiones para los escolares analizados como un único grupo, se presenta de forma diferente a las dimensiones identificadas por Verdugo y Sabeh en 2002, en estudio con niños argentinos y españoles. Cuando se indagó sobre lo que les hace sentirse felices, constataron que los argentinos de 8 años se refieren con mayor frecuencia al Ocio y Actividades Recreativas (46\%), seguido de las Relaciones Interpersonales (27\%) y Bienestar material (13\%). Los niños españoles de 8 años identifican también Ocio y Actividades Recreativas (49\%) con mayor frecuencia en relación a experiencias de 
satisfacción, seguidas así mismo de Relaciones Interpersonales (33\%) y a continuación, el Rendimiento y Bienestar material, ambas con $9 \%$ das respuestas.

Se destaca la divergencia en la percepción de los niños españoles y argentinos con respecto a los brasileros, pues el Rendimiento Escolar que se presenta como dimensión relevante para los brasileros (independientemente del tipo de escuela y por tanto, de la clase social) no se constituye en un factor tan importante para los escolares de la muestra estudiada en Argentina y España, mientras que la dimensión Ocio e Actividades Recreativas, que se mostró muy significativa para niños españoles y argentinos, no fue igualmente relevante para los escolares participantes en el nordeste de Brasil. Estudios posteriores, fundamentalmente de tipo cualitativo, podrían profundizar la dimensión Rendimiento Escolar que, aunque sea declarada entre las más relevantes, no mostró correlación con la calidad de vida de los escolares participantes de este estudio.

Es interesante observar la diferencia considerable en la escala de relevancia de las dimensiones entre la escuela públicas y privada cuando son analizadas separadamente. En la escuela particular los niños, que ya disfrutan de una vida saludable y con sus necesidades básicas atendidas, no dan tanta importancia al Bienestar Material, Físico y Emocional como los de la escuela pública.

Sin embargo, la dimensión Bienestar Colectivo y Valores assumió la última posición en la escuela pública, hecho que lleva a reflexionar sobre la interacción ecosistémica en la calidad de vida. Cómo podrían los pequeños proyectarse hacia el bienestar de la sociedad en general, cuando ésta no les garantiza condiciones mínimas necesarias para su propio Bienestar? También representa un punto de reflexión para el trabajo educativo en la escuela, en la construcción de ambientes favorables a la comunidad escolar, que prioricen los derechos y la inclusión social, como parte de la calidad de vida.

Es necesario percibir que las percepciones de los niños realmente varían de acuerdo al grupo social del cual forma parte en la sociedad y se modifican en relación con la cultura y valores sociales. Estos resultados permiten evaluar lo que es relevante desde la óptica de la propia persona, en este caso el escolar, y dar valor en la planificación y gestión escolar a lo que él considera importante para mejorar sua vida, con un enfoque de Planificación Centrada en la Persona (SCHALOCK, VERDUGO, 2003).

Así, por ejemplo, se fueran sugeridas actividades de intervención para mejoría de la Calidad de vida de estos escolares, las acciones no podrían ser las mismas, pues para la escuela pública lo ideal es que ellas estuvieran vinculadas a trabajar el Bienestar Físico y Emocional y el Rendimiento. Sin embargo, en la escuela particular sería necesario intervenir 
sobre las Relaciones Interpersonales, el Rendimiento y desarrollar acciones para mejorar el Bienestar Colectivo y Valores.

En estudios futuros, se sugiere la utilización de la conceptualización de Calidad de Vida en la infancia establecida por Sabeh, Verdugo y Prieto (2006), el estudio de CV en la escuela de niños con Necesidades Educacionales Especiales y el estudio de la calidad de Vida en estudiantes universitarios con Necesidades Educacionales Especiales, ampliando a la Educación Superior el concepto de calidad de vida en la escuela y de Escuela Promotora de la Salud.

\title{
A QUALIDADE DE VIDA INFANTIL E SUAS DIMENSÕES: PERCEPÇÃO DE ESCOLARES
}

\begin{abstract}
Resumo
O estudo objetiva avaliar a Qualidade de Vida (QV) percebida por escolares, identificar a relevancia de suas dimensões desde a óptica de crianças da rede pública e particular de ensino em Fortaleza-CE, e determinar se a dimensão Rendimento Escolar se relaciona com a QV. Participaram 90 crianças, entre oito e onze anos, que realizaram duas tarefas: questionário AUQEI (Manificat \& Dazord, 1997) e ordenamento de dimensões de QV. Também foi correlacionado o rendimento escolar com a pontuação final do AUQUEI. Constatou-se que a QV das crianças foi satisfatória ainda que com diferença significativa a favor dos alunos da escola particular, as dimensões mais relevantes para todos os estudantes foram: Rendimento, Bem estar físico e Emocional e Relações interpessoais, com diferenças ao analisar as preferencias por tipo de escola; não houve relação entre o Rendimento escolar e a pontuação final de QV.
\end{abstract}

Palavras-chave: Qualidade de vida. Criança. Escola

\section{THE QUALITY OF CHILDREN' S LIFE AND ITS DIMENSIONS: STUDENTS' PERCEPTION}

\begin{abstract}
The study aims to evaluate the Quality of Life (QL) perceived by students, to identify the relevance of its dimensions through the angle of children from public and private schools in Fortaleza-CE, and to determine if the dimension Learning Assessment relates to QL. 90 children participated, whose ages very from eigth to eleven, who performed two tasks: questionnaire AUQUEI (Manificat \& Dazord, 1997) and QL dimensions arrangement. Also the learning assessment was co-related to the final punctuation of AUQUEI. It was observed that the children's QL was satisfactory even with the significant difference in the private schools students' favor. The most relevant dimensions to all students were: Learning Assessment, Physical and Emotional Wellness and Interpersonal Relations, with differences when analyzing the preferences per type of school. There was no relation between the Learning Assessment and the final punctuation of QL.
\end{abstract}


Keywords: Quality of life. Children. School

\section{REFERÊNCIAS}

ASSUMPÇÃO, F. B. J. et al. Escala de avaliação da qualidade de vida (Autoquestionnaire qualité de vie enfant imagé): validade e confiabilidade de uma escala para qualidade de vida em crianças de 4 a 12 anos. Arq Neuropsiquiatr, São Paulo, v. 58, n.1, p. 119-127, nov. 2000.

BRASIL, T. B.; FERRIANI, V. P. L.; MACHADO, C. M. C. Inquérito sobre qualidade de vida relacionada a saúde em crianças e adolescentes portadores de artrites idiopáticas juvenis. Jornal de Pediatria, Porto Alegre, V.79, n.1, p.63-69. 2003.

DANTAS, R. A. S.; SAWADA, N. O.; MALERBO, M. B. Pesquisa sobre qualidade devida: revisão da produção científica das universidades públicas do estado de São Paulo. Revista Latino Americana de Enfermagem, Ribeirão Preto, v. 11, n.4, p.532-538, jul/ago. 2003.

EISER, C.; MORSE, R. Quality-of-life measures in chronic diseases of childhood. Health Technology Assessment. Sheffield, v. 5, n. 4, p.1-156. 2001.

Uma revisão de medidas de qualidade de vida para crianças com doenças crônicas. Arch Dis Child. v. 84, p. 205-211, fev. 2001.

EISER, C.; EISER J. R.; STRIDE C. B. Quality of life in children newly diagnosis with câncer and their mothers. Health and Quality of Life Outcomes. BioMed Central Ltd. , 2005. Retirado em: <htpp://www.hglo.com/content./3/1/29> Acesso em: 25 out. 2005.

FIALHO, L. F.; VALDÉS, M. T M. Qualidade de vida na escola: e a promoção de ambientes favoráveis à saúde. In: ENCONTRO DE PÓS-GRADUAÇÃO E PESQUISA DA UNIFOR, $\mathrm{n}^{\circ}$ 6, 2006, Fortaleza. VI encontro de pós-graduação e pesquisa da UNIFOR. Universidade de Fortaleza, 2006. p.15.

GÓMEZ- VELA, M.; SABEH, E. N. Calidad de Vida. Evolución del Concepto y su Influencia en la Investigación y la Práctica. Disponível em: $<$ http://www3.usal.es/ inico/investigacion/invesinico/calidad.html> Acessado em: 17 mai 2004

GÓMEZ- VELA, M.; VERDUGO, M. A. Evaluación de la calidad de la vida de alumnos de educación secundaria obligatoria con necessidades especiales y sin ellas. In: Apoyos, autodeterminación y calidad de vida: Actas de las IV Jornadas Científicas de Investigación sobre personas con discapacidad, n. 4, 2003, Salamanca: Amarú Ediciones, 2003. p. 482-496.

GUZZO, R. S. L. Saúde psicológica, sucesso escolar e eficácia da escola: desafios do novo milênio para a psicologia escolar. In: PRETTE, Z. A. P. D. (org.). Psicologia Escolar e Educacional: Saúde e Qualidade de Vida. Campinas, SP: Editora Alínea, 2003. p. .25-42.

Harding, L. Children's quality of life assessments. A review of generis and health related quality of life measures completed by children and adolescents. Clinical Psychology \& Psychoterapy. Volume 8, Issue 2, 2001, P. 79-96 
JOKOVICH, A.; LOCKER, D.; GUYATT, G. How well do parents know their children. Implications for proxy reporting of child health-related quality of life. Quality of life research. v.13, p.1297-1307. 2004.

MANIFICARD, S.; DAZORD, A. Évaluation de la qualité de vie de I"enfant: validation d"un questionnaire, premiers resultants. Neuropsychiatr Enfance Adolesc, v. 45, p.106-114. 1997.

MINAYO, M. C. S.; HARTZ, Z. M. A. ; BUSS, P. M. Qualidade de vida e saúde: um debate necessário. Ciência \& Saúde Coletiva, Rio de Janeiro, v.5, n.1, p.7-18. 2000.

PREBIANCHI, H. B. Medidas de qualidade de vida para crianças: aspectos conceituais e metodológicos. Psicologia teoria e prática, v. 5, n. 1, p.57-69. 2003.

RAJMIL, L. et al. Calidad de Vida relacionada con la Salud (CRVS) en la infancia y la adolescencia: revisión de la bibliografía y de los instrumentos adaptados en España. Gac Sanit, v. 15, n. 4, p. 34-43. 2004.

SABEH, E. N. et al. Evaluación de calidad de vida en niños de escolaridad primaria. In: Verdugo, M. A.; Jordán de Urríes, F. B. Apoyos, autodeterminación y calidad de vida: Actas de las V Jornadas Científicas de Investigación sobre Discapacidad. Salamanca: Amarú Ediciones, 2003.

SABEH, E. N.; VERDUGO, M.; PRIETO, G. A. Dimensiones e indicadores de la calidad de vida en la infancia. In: VERDUGO, M. A. (dir.). Cómo mejorar la calidad de vida de las personas con discapacidad: Instrumentos y estratégias de evaluación. Salamanca: Amarú, 2006. p.61-76.

SCHALOCK, R.; VERDUGO, M. A. Calidad de vida: manual para profesionales de la educación, salud y servicios sociales. Madrid: Alianza Editorial, 2003.

VERDUGO, M. A.; SABEH, E. N. Evaluación de la percepción de calidad de vida en la infancia. Psicothema, Salamanca, v. 14, n.1, p. 86-91, jun. 2002.

WHITE-KONING, M. L. et al. Asssessment of response competence before administering self-reported quality of life instruments to children whit cerebral palsy and intellectual impairment. Dev. Med. Child Neurol, n. 47, p. 47-61. 2005.

Data de recebimento: 21/08/14

Data de aceite: $28 / 08 / 16$

\section{Sobre os autores:}

Lia Machado Fiuza Fialho é Professora adjunta do Centro de Educação da Universidade Estadual do Ceará - UECE. Pós-doutora em Educação pela Universidade Federal da Paraíba UFPB. Doutora em Educação Brasileira pela Universidade Federal do Ceará - UFC. Mestre em Saúde Coletiva pela Universidade de Fortaleza - UNIFOR. Especialista em Inclusão da Criança Especial no Sistema Regular de Ensino pela UFC, em Psicologia da Educação FLATED e em Psicopedagogia Institucional e Clínica - FLATED. Pedagoga pela Universidade Estadual do Ceará - UECE. Editora chefe da coleção Práticas Educativas EdUECE. Endereço eletrônico: lia_fialho@yahoo.com.br 
José Rogério Santana é Possui graduação em pedagogia pela Universidade Federal do Ceará (UFC), mestrado acadêmico e doutorado em Educação pela Universidade Federal do Ceará (UFC), Pós-doutorado em História da Educação no Programa de Pós-graduação da Universidade Federal da Paraíba (UFPB). É professor adjunto da Universidade Federal do Ceará. Endereço eletrônico: rogerio@ virtual.ufc.br

Cristine Brandenburg é Mestranda pelo Programa de Pós Graduação em Educação Brasileira pela Universidade Federal do Ceará, Bolsista CNPq. Possui graduação em Fisioterapia pela Universidade de Santa Cruz do Sul (2008). Endereço eletrônico: crisfisio13@ gmail.com 